\title{
The carotenoids of Pouteria campechiana (sinhala: ratalawulu)
}

\author{
Maduka S. de Lanerolle ${ }^{1 *}$, A. M. Buddhika Priyadarshani ${ }^{2}$, D. B. Sumithraarachchi ${ }^{3}$ and E. R. Jansz $^{4}$ \\ 45/1, St. Mary's Road, Wattala. \\ 2 Department of Biochemistry, University of Sri Jayewardenepura, Gangodawila, Nugegoda. \\ 3 14/B5/37 Hantane New Town, Hantane. \\ 4 29/1, Pietersz Place, Nugegoda.
}

\begin{abstract}
The yellow fruit lavalu found in Sri Lanka has been confirmed by taxonomical studies as Pouteria campechiana (Sinhala: Ratalawulu). This is the only yellow lavalu found in Sri Lanka and is not of the Chrysophyllum species. The leaf, fruit and seed characteristics were studied and compared with herbarium specimens at the National Herbarium. The carotenoids were dominated by neoxanthin. Total carotenoid content was high and varied from 1.9 to $23.5 \mathrm{mg} . \mathrm{g}^{-1}$ dry weight (DW). Individual carotenoid concentrations varied markedly from specimen to specimen ( $\beta$-carotene, from traces to 156 , $\zeta$-carotene, trace amounts, $\beta$-cryptoxanthin, from traces to 1106, violaxanthin, from less than 188 to 1151 , neoxanthin, from 1594 to 19,270 , unidentified I, from traces to 627 , unidentified II, from 68 to $\left.1162, \mu \mathrm{g} \cdot \mathrm{g}^{-1} \mathrm{DW}\right)$. Due to the presence of a carbohydrate gum the normal extraction procedure for carotenoids had to be modified. Identification was done after separating by open column chromatography (OCC) by using visible spectral data, chemical tests, thin layer chromatography (TLC) as well as high performance liquid chromatography (HPLC). Quantification was mainly carried out by HPLC using $\beta$-apo-8'-carotenal as the internal standard. Results showed two extremes of retinol equivalents (RE) in Pouteria campechiana (traces and 759 to $11,813 \mathrm{RE} .100 \mathrm{~g}^{-1} \mathrm{DW}$ ). These ranges as well as the varying shapes of fruit are indicators that the trees are extremely hybridized. However, its carotenoid profile and high colour intensity could give rise to antioxidant properties and also find use as a natural food colourant as it has an unusually high concentration of total carotenoids.
\end{abstract}

Keywords: Carotenoids, Pouteria campechiana, ratalawulu, Sri Lanka

\section{INTRODUCTION}

Chrysophyllum roxburghii (synonym C. lanceolatum), C. oliviforme (synonym C. monopyrenum), C. cainito and Pouteria campechiana are all referred to as "lavalu" in Sinhala. Pouteria campechiana have yellow fruits while all the above Chrysophyllum species in Sri Lanka do not have yellow fruits ${ }^{1}$. P. campechiana is common in Sri Lanka, especially in home gardens. There is a disagreement regarding the value of the different species as pro-vitamin A sources. Rodriguez-Amaya, (personal communication) in Phillipines states that the retinol equivalent is high, whereas Chandrika et al. (2005) and Chandrika (2004) states that the retinol equivalent is low; $68 \mathrm{RE} / 100 \mathrm{~g}$, and $23 \mathrm{RE} / 100 \mathrm{~g}$ respectively. Past studies on lavalu have identified the specimens as that of C. roxburghii ${ }^{2-4}$. However, the descriptions of the fruit ${ }^{2-4}$ and photographs ${ }^{4}$ do not match with those of C. roxburghii identification. The fruit pulp of $C$. roxburghii is white ${ }^{1}$ in colour and has negligible carotenoid content. The lavulu fruits for analysis in the present study were collected from home gardens in Sri Lanka, which according to Verdcourt and Meijer" is called "Ratalawulu", has 1-6 seeds and was introduced to Sri Lanka from Central or South America. The objectives of this study were to: (i) confirm the identity of the common home garden lavalu using taxonomic characteristics and (ii) study the carotenoid profile of the fruits with a view to determining the pro-vitamin A potential.

\section{METHODS AND MATERIALS}

Plant material: Specimens of fruits, leaves and when possible inflorescences were collected from home gardens in 1.Kadawata, 2.Kurunegala, 3.Maharagama, 4.Makola, 5.Peradeniya and 6.Nawala (These specimen numbers are used in the text). Ripe fruits were deepfrozen at $-20^{\circ} \mathrm{C}$ and analyzed $2-3 \mathrm{~d}$ apart. On the day of analysis the following parameters were measured: (1) fruit dimensions, (2) shape of fruit, (3) weight of fruit, (4) weight of edible pulp, (5) number and characteristics of seeds.

\footnotetext{
${ }^{*}$ Corresponding author
} 
Taxonomy: Plant specimens were examined by a phytotaxonomist for venation, shape and margin of leaf and characteristics of fruit and compared with data of Verdcourt and Meijer ${ }^{1}$, Barlow ${ }^{5}$ as well as specimens in the National Herbarium, Peradeniya.

Moisture Content: This was determined in triplicate by freeze-drying $1 \mathrm{~g}$ samples to constant weight. This method gave results similar to the Dean and Stark method ${ }^{6}$.

Extraction: Extraction of the yellow sticky fruit pulp (2-5 g) following the standard extraction procedures using cold acetone $^{7}$ was difficult and filtration by suction was also difficult due to the presence of a gum. Extraction was not possible in the specimens where the content of gum was very high. The problem necessitated a modification in the extraction procedure. To dissolve the gum in water before extraction of carotenoids, a known weight (2-5 g) of the pulp was collected into a centrifuge tube and vortexed with distilled water $(30 \mathrm{~mL})$, followed by centrifugation ( $25000 \mathrm{rpm}$ for $15 \mathrm{~min}$ ). The supernatant was decanted. After mixing the pellet with water, the centrifugation was repeated depending on the extent of gum. The extent of gum in the water extract could be assessed by precipitation with acetone. The rest of the procedure was as previously described ${ }^{7}$. The carotenoids of the acetone extract was partitioned to petroleum ether containing 2 to $4 \%$ diethyl ether (diethyl ether concentration was increased due to the concentrations of the hydroxy carotenoids being high in lavalu ${ }^{3,4}$ ). Saponification was done with $10 \%$ methanolic $\mathrm{KOH}$ as the pulp contained fatty acid derivatives of hydroxy carotenoids as indicated by the results of preliminary open column chromatography (OCC). The petroleum ether was evaporated using a stream of nitrogen. It is to be noted that the entire carotenoid isolation, separation and identification procedures were carried out under dim light and where possible in a nitrogen atmosphere to reduce the impact of atmospheric oxygen and light and thereby preventing isomerisation and photodegradation.

Open column chromatography (OCC): The extract was subject to $\mathrm{MgO}$ :Celite(1:1) OCC and eluted with a gradient of petroleum ether and acetone ${ }^{7}$.

Identification of carotenoids: Each colour band emerging was concentrated by flushing nitrogen gas in to the sample to remove the solvent; it was then subjected to scanning by double beam spectrophotometry (Shimadzu, UV-1601 model). Identification was done by standard methods of Rodriguez- Amaya ${ }^{7}$.

Quantification: This was carried out by two methods, (1) the formula of Rodriguez- Amaya ${ }^{7}$ using absorbance of the isolates eluted from OCC and by (2) Reverse Phase (RP) HPLC using the internal standard $\beta$-apo-8'-carotenal.

\section{RESULTS}

\section{Taxonomy}

Leaves from trees where fruits were collected were compared with those of an authentic specimen of $C$. roxburghii and were observed to be different. The species was confirmed as $P$. campechiana, by comparing data of Verdcourt and Meijer ${ }^{1}$, Barlow ${ }^{5}$ as well as specimens in the National Herbarium, Peradeniya.

All fruits had the orange/yellow colour typical of fruits of $P$. campechiana on the surface, as well as in the pulp. However, there were variations in fruit shape: typical pyriform, as well as mammariform, broadly globose ovoid and oblong ovoid indicating extensive hybridization as in the case of many common fruits such as papaw. Other criteria include the extent of carbohydrate gum and the number of seeds in each fruit from the same tree (e.g. 1 seed), compared to those of other trees (2 4 seeds). These criteria are now supported by differing carotenoid profiles of specimens from different trees.

\section{Modification of extraction procedure}

The pulp had to be extracted with water. The aqueous supernatant contained an acetone precipitable gum, which hindered filtration of the direct acetone extract. This caused a thick mat being formed enclosing the carotenoids during the normal acetone extraction. Water extraction removed the gum and subsequent acetone extraction was easy. The gum content was very high in specimens 2 and 6 ( 2 and multi-seeded) as evidenced by the number of water extractions needed, the poor viscosity of the water extract, and the extent of precipitation of gum from water extract by acetone (Carotenoids of samples 2 and 6 were not extracted at all without the water extraction). On adding acetone to the water extract a watery gummy precipitate was obtained which had earlier been identified as a carbohydrate gum ${ }^{2}$.

\section{Moisture content}

Moisture content varied from 58.6 to $69.9 \%$.

\section{Carotenoid profile}

As determined by OCC, $\beta$-carotene and $\beta$-cryptoxanthin were the only pro-vitamin A carotenoids and violaxanthin, neoxanthin, $\zeta$-carotene, unidentified fractions $\mathrm{I}$ and II were non-provitamin A carotenoids. According to 
Table 1: Carotenoid content and retinol equivalent (RE) of lavulu

\begin{tabular}{|c|c|c|c|c|c|c|}
\hline \multirow{2}{*}{$\begin{array}{l}\text { Carotenoid } \\
\left(\mu \mathrm{g} . \mathrm{g}-{ }^{1} \mathrm{DW}\right)\end{array}$} & \multicolumn{6}{|c|}{ Specimen No: } \\
\hline & 1 & 2 & 3 & 4 & 5 & 6 \\
\hline$\beta$-Carotene & $\operatorname{Tr}$ & $\operatorname{Tr}$ & $\operatorname{Tr}$ & $\operatorname{Tr}$ & 156 & $\operatorname{Tr}$ \\
\hline$\beta$-Cryptoxanthin & 91 & 475 & (188* & $\operatorname{Tr}$ & 1106 & 520 \\
\hline Violaxanthin & 103 & 683 & \{ & 29 & 1151 & 703 \\
\hline Neoxanthin & 1594 & 8077 & 3020 & 3946 & 19,270 & 15,308 \\
\hline Unidentified I & 11 & $\operatorname{Tr}$ & $\operatorname{Tr}$ & $\operatorname{Tr}$ & 628 & $\operatorname{Tr}$ \\
\hline Unidentified II & 68 & 163 & 229 & 319 & 1162 & 572 \\
\hline$\zeta$-Carotene & $\mathrm{ND}$ & ND & ND & ND & ND & ND \\
\hline RE .100-1gDW & 759 & 3962 & $\begin{array}{l}\text { Not } \\
\text { calcu }\end{array}$ & $\operatorname{Tr}$ & 11813 & 4337 \\
\hline
\end{tabular}

- Specimen numbers 1 to 6 is as given in text (plant material)

- Quantification was done by HPLC

- Each of the 6 samples were analysed in duplicate

- $\quad \mathrm{ND}=$ Not detected $(\zeta$-carotene was not detected at $450 \mathrm{~nm})$

- $\mathrm{RE}$ was calculated from $6 \mu \mathrm{g}$ of $\beta$-carotene $=1 \mu \mathrm{g}$ retinol and $12 \mu \mathrm{g}$ of $\beta$ - cryptoxanthin $=1 \mu \mathrm{g}$ retinol

- $\quad * \beta$-Cryptoxanthin and violaxanthin contents were added as they were co-chromatographed in sample No: 3

- $\operatorname{Tr}=$ Trace amount

- $\quad \mathrm{DW}=$ Dry weight

the chromatographic behaviour on TLC, unidentified fraction I had similar chemical and TLC characteristics to that of neoxanthin but was not neoxanthin and unidentified fraction II could be $\beta$-cryptoxanthin mono epoxide, but this could not be confirmed due to impurities. Therefore unidentified fraction II was not counted in calculating

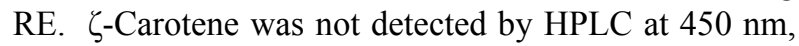
as its concentration was very low $\left(\lambda_{\max }\right.$ of $\zeta$-carotene is $400 \mathrm{~nm}$ ). Deviations, as expected were large and therefore calculation of standard deviations (SD) were not possible. RE was very variable and only specimen No: 5 had significant $\beta$-carotene content. Two samples (specimen No: 3 and 4) indicated co-chromatography of $\beta$-cryptoxanthin and violaxanthin in HPLC but OCC showed no $\beta$-cryptoxanthin in sample No:4. According to the present study $\beta$-cryptoxanthin is the only consistent contributor to the RE.

\section{DISCUSSION}

Detailed taxonomic examination confirmed that the common home garden lavalu (ratalawulu) in Sri Lanka is P. campechiana. The specimens collected had 1,2, 3 and 4 seeds, which corresponded to the 1 to 6 seed description in an authoritative source ${ }^{1}$. The variations in leaves, shape of fruits and extent of gum were seen, indicating that there may be sub-species ${ }^{1}$ or hybridization. However, this has to be confirmed by DNA marker studies.
The two and multi-seeded specimens had much more gum and this brings into question the validity of the results of past studies ${ }^{3,4}$ on multi-seeded types (5 8 seeds), which from photographs ${ }^{4}$ and description ${ }^{3}$ is no doubt on the same species as this study. The doubt is caused because the water extraction step to remove the gum has not been used in order to ensure complete extraction of carotenoids.

That the earlier studies ${ }^{2-4}$ had not used the very rare wild species of $C$. roxburghii (which is also known as "koholla lavalu" due to excessive white sap) was clear from descriptions ${ }^{2-4}$ and photographs ${ }^{4}$. This was confirmed by collection of a specimen of $C$. roxburghii and comparing it with herbarium specimens with respect to morphology of leaves and flowers. It was also confirmed that the fruit pulp of $C$. roxburghii was white and its fruit did not correspond to the yellow pulp shown in photographs ${ }^{4}$. The specimens of Pouteria campechiana of this study corresponded to the description of Verdcourt and Meijer ${ }^{1}$. Another important difference was that the earlier study ${ }^{3,4}$ found violaxanthin as the dominant carotenoid. The present study showed that neoxanthin was by far the dominant carotenoid in all specimens. This conclusion was supported by the following. (i) epoxy furanoid shift (middle peak shift by $14 \mathrm{~nm}$ and average shift of three peaks by $13 \mathrm{~nm}$ for neoxanthin (one epoxy group). A middle peak shift by $39 \mathrm{~nm}$ and 
average shift of three peaks by $41 \mathrm{~nm}$ for violaxanthin (two epoxy groups which was also detected in the study) (ii) the blue colour in the fuming $\mathrm{HCl}$ test for neoxanthin and green for violaxanthin (iii) $\mathrm{R}_{\mathrm{f}}$ values were 0.25 and 0.22 for violaxanthin ( 2 hydroxy groups) and neoxanthin (3 hydroxy groups) respectively. The above shows that the identification of these two were correct in the present study. RE was very variable; one specimen gave only a trace amount while in four others the content ranged from 759 to $11813.100 \mathrm{~g}^{-1}$ dry weight (DW). In calculation of $\mathrm{RE}$, two previous reports ${ }^{3,4}$ calculating on the basis of the same results gave RE as 68 and 23, respectively.

Previous studies ${ }^{3,4}$ had not taken into account the provitamin A carotenoids: $\beta$-cryptoxanthin and its epoxide. In this study $\beta$-cryptoxanthin was the major contributor to RE. Further, unidentified II could be $\beta$-cryptoxanthin5'6'-epoxide (also a pro-vitamin A carotenoid), which would increase RE further. This was not taken into account in the study as there was an impurity detected in the compound on TLC. The present study showed that no correlation could be obtained from taxonomy on one hand and carotenoid profile on the other. That is, there was no chemotaxonomic correlation with regard to carotenoid profile. Therefore, a recommendation on pro-vitamin A potential based on taxonomy is not possible. The extent of carbohydrate ${ }^{2}$ gum correlated with the number of seeds (consistently $>1$ ) being higher than for specimens of the mono-seeded type. Variety and environmental effects may be involved in a marked variation of shape of fruit, carotenoid content and RE as seen in the present study. However, this was most probably due to extensive hybridization. Verdcourt and Meijer ${ }^{1}$ mentioned two varieties of $P$. campechiana but the specimens of this study could not be sub-divided on the basis detailed by that authority. The total carotenoid content was much higher than that of other sources (1.9 to $23.5 \mathrm{mg} . \mathrm{g}^{-1} \mathrm{DW}$ ). Therefore the deep yellow fat-soluble colour could lend itself as a good food colour for fat based foods such as margarine. The structures of the carotenoids indicate that antioxidant potential of Ratalawulu could be high if the oxygenated carotenoids are absorbable.

\section{CONCLUSION}

There appear to be two extremes of RE in P. campechiana (traces and 759 to 11,813 RE. $100 \mathrm{~g}^{-1} \mathrm{DW}$ ). The major contributor to RE was $\beta$-cryptoxanthin. However there was no correlation between taxonomical characteristics of leaf, fruit and seed with total carotenoids, neoxanthin, $\beta$-cryptoxanthin and RE making it impossible to pinpoint which Ratalavalu specimens are good sources of pro-vitamin A carotenoids based on morphological characteristics.

\section{Acknowledgement}

The authors thank IPICS grant SRI:07 for financial support.

\section{References}

1. Verdcourt B. \& Meijer W. (1995). A revised Handbook to Flora of Ceylon, Volume IX, (Eds. Dassanayake, Fosberg \& Clayton). Oxford and IBH Press, New Delhi, Bombay, Calcutta.

2. Abeysekara M.(1993). Studies on betalains in Beta vulgaris and carotenoids and carbohydrates in Chrysophyllum roxburghii fruit. Ph.D. thesis, University of Colombo, Colombo 03.

3. Chandrika U.G., Jansz E.R. \& Warnasuriya N.D. (2005). Identification and HPLC quantification of carotenoids of the fruit pulp of Chrysophyllum roxburghii. Journal of the National Science Foundation of Sri Lanka 33(2): 93-98.

4. Chandrika U.G. (2004). Content, bioavailability, bioconversion and antioxidant activity of carotenoids of some Sri Lankan fruits and green leafy vegetables. Ph.D. thesis, University of Sri Jayewardenepura. Ganagodawila, Nugegoda.

5. Barlow H.F. (1991). Macmillans Tropical Planting and Gardening, sixth edition. (Revised by H.S. Barlo, I. Enoch \& R.A. Russell) Malayan Nature Society, Kuala Lumpur, Malaysia.

6. Association of Official Analytical Chemists (1984). Official methods of analysis of the AOAC, Fourteenth edition, AOAC, Washington, DC.

7. Rodriguez-Amaya D.B. (1999). A Guide to Carotenoid Analysis in Foods. ILSI Press, Washington, USA. 